\title{
AN ANALYTIC-NUMERICAL METHOD OF COMPUTATION OF THE LIAPUNOV AND PERIOD CONSTANTS DERIVED FROM THEIR ALGEBRAIC STRUCTURE. *
}

\author{
ARMENGOL GASULL ${ }^{\dagger}$, ANTONI GUILLAMON ${ }^{\ddagger}$, AND VÍCTOR MAÑOSA $\S$
}

Key words. centre point, Liapunov constants, isochronicity, analytic-numerical method.

AMS subject classifications. 65L07, 34D20; 34C25.

Abstract. We consider the problem of computing the Liapunov and the period constants for a smooth differential equation with a non degenerate critical point. First, we investigate the structure of both constants when they are regarded as polynomials on the coefficients of the differential equation. Secondly, we take advantadge of this structure to derive a method to obtain the explicit expression of the above-mentioned constants. Although this method is based on the use of the Runge-KuttaFehlberg methods of orders 7 and 8 and the use of Richardson's extrapolation, it provides the real expression for these constants.

1. INTRODUCTION AND MAIN RESULTS. In this paper we deal with the problem of computing the Liapunov and period constants for the next type of differential equations:

$$
\left\{\begin{array}{l}
\dot{x}=-y+P(x, y) \\
\dot{y}=x+Q(x, y)
\end{array}\right.
$$

where $P(x, y)$ and $Q(x, y)$ are analytic functions in a neighbourhood of the origin, and begin, at least, with second order terms. These systems can be expressed in the complex plane using the following notation:

$$
\dot{z}=i z+F(z, \bar{z})
$$

where $F(z, \bar{z})=\sum_{k>2} F_{k}(z, \bar{z}), F_{k}(z, \bar{z})=\sum_{j=0}^{k} f_{k-j, j} z^{k-j} \bar{z}^{j}, f_{k-j, j} \in \mathbf{C}$, and the dot indicates the derivative with respect to $t$, with $t \in \mathbf{R}$.

The problem of determining whether (1) has a center or a focus at the origin can be solved by studying the Poincaré return map. This study can be done (using the power series of the return map) by means of the computation of infinitely many real

\footnotetext{
* Partialy supported by the DGICYT grant number PB93-0860.

$\dagger$ Departament de Matemàtiques, Universitat Autònoma de Barcelona, Edifici Cc 08193 Bellaterra, Barcelona. Catalonia, Spain (gasullomat.uab.es)

† Departament de Matemàtica Aplicada I, Universitat Politècnica de Catalunya, Dr. Marañón 44-50, 08028 Barcelona. Catalonia, Spain (toni @ma1.upc.es)

$\S$ Departament de Matemàtica Aplicada III, Universitat Politècnica de Catalunya, Colom 1, 08222 Terrassa, Barcelona. Catalonia, Spain (manosa@ma3.upc.es)
} 
numbers, $v_{2 m+1}, m \geq 1$, called the Liapunov constants. In fact, we have that if for some $m, v_{3}=v_{5}=\cdots=v_{2 m-1}=0$, and $v_{2 m+1} \neq 0$, then the origin is a focus of which the stability is determined by the sign of $v_{2 m+1}$, while if all $v_{2 m+1}$ are zero, then the origin is a center, see for instance [1].

A closely related problem is the following: assume that (1) has a center at the origin and consider the period of all its periodic orbits. The origin of (1) is an isochronous center if and only if the period is independent of the orbit. When is the origin of (1) an isochronous center? It turns out that the solution to this problem can be obtained (using the power series of the period function) by computing infinitely many real numbers, $P_{2 m}, m \geq 1$, called the period constants and by forcing them all vanish.

In the case that (1) is a family of polynomial differential equations, the constants are functions in the coefficients of the system. Moreover, in the case that (1) is a polynomial family of differential equations of fixed degree, the Hilbert Basis Theorem implies that a finite number of Liapunov constants vanishing is enough to give the characterization of the centers of the family, just as a finite number of Liapunov and period constants vanishing is enough to characterize the isochronous centers of the family.

Many authors have dealt with the problem of computing the constants. Without being exhaustive, see for instance [3], [4], [6], [7], [9], [10], [14] or [16]. All the approaches in order to calculate the constants involve a lot of computations. In order to have an a priori estimation of the complexity of the result, it is very useful to know properties of the Liapunov and period constants when they are considered as functions of the coefficients of (1).

Before stating our results we need some definitions.

We say that $M$ is a monomial of (1) when $M=\prod_{k, l} f_{k, l}^{m_{k, l}}{\overline{f_{k, l}}}^{n_{k, l}}$, with $m_{k, l}, n_{k, l} \in$ $\mathrm{N}$, where the product is finite, and $f_{k, l}$ is any coefficient of $F_{k+l}(z, \bar{z})$.

Let $v_{2 m+1}$ be the $m^{\text {th }}$ Liapunov constant (respectively, let $P_{2 m}$ be the $m^{\text {th }}$ period constant). We will also say that a monomial of (1), $M$, is a monomial of $v_{2 m+1}$ (or of $\left.P_{2 m}\right)$, if either $\operatorname{Re}(M)$ or $\operatorname{Im}(M)$ appear in the expression of the constant.

We define the degree, $\operatorname{deg}(M)$, the quasi-degree, $\operatorname{qd}(M)$, and the weight of $M$, $\mathrm{w}(M)$, respectively, as:

$$
\begin{gathered}
\operatorname{deg}(M)=\sum_{k, l}\left(m_{k l}+n_{k l}\right), \quad \mathrm{qd}(M)=\sum_{k, l}(k+l-1)\left[m_{k l}+n_{k l}\right], \quad \text { and } \\
\mathrm{w}(M)=\sum_{k, l}(1-k+l)\left[m_{k l}-n_{k l}\right] .
\end{gathered}
$$

Finally, we say that a monomial of (1) of weight zero, $M$, is basic if $M^{\prime} \mid M$ and $\mathrm{w}\left(M^{\prime}\right)=0$ imply that $M^{\prime}= \pm M$. Roughly speaking, the basic monomials are the prime factors of the monomials of weight zero.

With the above notation, the following result is well-known, see [2], [17], [12], [13] and [18].

Theorem 1. Let $M$ be a monomial of $v_{2 m+1}$ or $P_{2 m}$. Then, $\operatorname{qd}(M)=2 m$ and 
$\mathrm{w}(M)=0$.

The property $\mathrm{w}(M)=0$ is derived from the fact that the constants are invariant under rotations of the vector field. Property $q d(M)=2 m$ comes from the effect of homoteties in the vector field.

In Theorem 1, it is given some information about the monomials that appear in the Liapunov and period constants. In our main result, we improve it by describing how these monomials are distributed according to their degrees.

Theorem A The following statements hold.

(i) Let $M_{1}, M_{2}, \cdots, M_{k}$ (respectively $M_{k+1}, M_{k+2}, \cdots, M_{k+l}$ ) be monomials of $v_{2 m+1}$ with even degree (respectively odd degree). Then:

$$
v_{2 m+1}=\sum_{i=1}^{k} \alpha_{i} \operatorname{Im}\left(M_{i}\right)+\sum_{i=k+1}^{k+l} \beta_{i} \operatorname{Re}\left(M_{i}\right) .
$$

for some $\alpha_{i}, \beta_{i} \in \mathbf{R}$.

(ii) Let $M_{1}, M_{2}, \cdots, M_{p}$ (respectively $M_{p+1}, M_{p+2}, \cdots, M_{p+q}$ ) be monomials of $P_{2 m}$ with even degree (respectively odd). Then:

$$
P_{2 m}=\sum_{i=1}^{p} \gamma_{i} \operatorname{Re}\left(M_{i}\right)+\sum_{i=p+1}^{p+q} \delta_{i} \operatorname{Im}\left(M_{i}\right) .
$$

for some $\gamma_{i}, \delta_{i} \in \mathbf{R}$.

The proof of Theorem A appears in Section 2. It is an improvement of Theorem 1 and of Theorem 2 of [2]. Observe that for any vector field, $v_{2 m+1}$ and $P_{2 m}$ are real numbers. Hence, if $M$ is a monomial of (1), $v_{2 m+1}=\alpha M+\bar{\alpha} \bar{M} N$, where $N$ denotes the sum of the other monomials appearing in its expression, and so, $v_{2 m+1}=2 \operatorname{Re}(\alpha) \operatorname{Re}(M)-2 \operatorname{Im}(\alpha) \operatorname{Im}(M)+N$ (an analogous argument is valid for $\left.P_{2 m}\right)$. Therefore, Theorem A reduces in one half the estimation of the length of the Liapunov and period constants obtained using only Theorem 1. To give an idea of this reduction, in Table 1 we display different estimations of the number of terms of $v_{2 m+1}$ and $P_{2 m}$, considered as polynomials on the monomials $M_{i}$. These estimations have been obtained by imposing progressively the following constraints:

Second column: $v_{2 m+1}$ and $P_{2 m}$ are real-valued polynomials of degree $2 m$.

Third column: The monomials of $v_{2 m+1}$ and $P_{2 m}$ satisfy the restrictions of Theorem 1.

Fourth column: The monomials of $v_{2 m+1}$ and $P_{2 m}$ satisfy the restrictions of Theorem 1 and Theorem A.

Fifth column: The actual number of monomials of $v_{2 m+1}$ or $P_{2 m}$ computed in $[6]$.

On the other hand, when we want to compute some exact Liapunov or period constant, from the above results we know that it is a polynomial in which the only unknowns are the coefficients of its monomials. It is also well-known that these coefficients are rational multiples of $\pi$ and so, multiplying by an appropriate factor, they can 
TABLE 1

Estimations of the number of monomials.

\begin{tabular}{|l|r|r|r|r|}
\hline Const. & $\begin{array}{r}\text { Polynomials } \\
\text { of deg }=2 m\end{array}$ & From Thm. 1 & $\begin{array}{r}\text { From Thms. 1 } \\
\text { and A }\end{array}$ & $\begin{array}{r}\text { Actual } \\
\text { number }\end{array}$ \\
\hline \hline$v_{3}$ & 119 & 7 & 2 & 2 \\
$v_{5}$ & 91.389 & 79 & 28 & 22 \\
$v_{7}$ & 156.238 .907 & & 310 & 259 \\
\hline$P_{2}$ & 119 & 7 & 5 & 4 \\
$P_{4}$ & 91.389 & 79 & 40 & 29 \\
\hline
\end{tabular}

be reduced to integer numbers. The way of computing these coefficients is a key point in our approach since we obtain them from numerical integrations of some particulars cases of the ordinary differential equation. The process, based on a combination of the Runge-Kutta-Fehlberg method with variable step and the Richardson's extrapolation method, is developed in Section 3. In contrast to most of the other methods to compute the Liapunov and period constants, it does not require the use of computer algebra systems. In our method we only need to list all the monomials of $v_{2 m+1}$ or $P_{2 m}$, choose appropriate systems that can provide their coefficients and carry out the numerical process to obtain them effectively. The last step consists only of solving a system of linear equations.

In Section 3.1, Theorem 3, we illustrate our approach by computing the Liapunov constant $v_{5}$ for a general system of type (1). In Section 3.2, as an example of how the numerical method works and how the round-off errors increase if it is applied to the computation of $v_{7}$, we apply our method to compute $v_{7}$ for a particular system. These computations are obtained by using artihmetics of double precision. In general, to compute higher order Liapunov or period constants, one should work with quadruple or even higher precision.

Finally, observe that in order to apply our method to a given polynomial system of the form (1), we need to know all their monomials of weight zero. To list them it would be useful to find a kind of "finite system of generators". In the Appendix we prove that those generators are the basic monomials defined above. In our opinion, the use of basic monomials in systems with few coefficients provides an elegant and compact form to present the Liapunov (and the period) constants (see, for instance, Remark 4 in the Appendix).

Acknowledgement. We are very grateful to Francesc Mañosas for the stimulating discussions and his ideas to prove the results of the Appendix.

2. PROOF OF THEOREM A. We need a preliminary result and some notation.

Definitions. We will denote by

(i) $\mathcal{P}_{0}$ the set of functions of the form $\operatorname{Re}(P(\theta)$ ), where $P(\theta)$ writes as

$$
P(\theta)=\sum_{l} p_{l}(\theta) M_{l} i^{\operatorname{deg}\left(M_{l}\right)}
$$


$M_{l}$ denotes any monomial of $(1)$, and $p_{l}(\theta)$ are trigonometric polynomials with real coefficients, that is, elements of $\mathbf{R}\left[e^{i \theta}\right]$.

(ii) $\mathcal{P}_{1}$ the set of functions of the form $\operatorname{Re}(i P(\theta))$, such that $\operatorname{Re}(P(\theta)) \in \mathcal{P}_{0}$.

When we have an operation ' $*$ ' between the elements of two sets $A$ and $B$, we denote $A * B=\{a * b: a \in A, b \in B\}$.

LEMMA 2. The following relations are satisfied.

(i) $\mathcal{P}_{0}+\mathcal{P}_{0} \subset \mathcal{P}_{0}, \mathcal{P}_{1}+\mathcal{P}_{1} \subset \mathcal{P}_{1}$

(ii) $\mathcal{P}_{i} \cdot \mathcal{P}_{j} \subset \mathcal{P}_{i+j}$, where $i, j \in\left(\mathrm{Z}_{2},+\right)$.

Proof. The proof of (i) is trivial. Here we proof for instance the case $\mathcal{P}_{0} \cdot \mathcal{P}_{0} \subset \mathcal{P}_{0}$ of (ii).

Consider $a(\theta), b(\theta) \in \mathcal{P}_{0}$, that is:

$$
\begin{aligned}
& a(\theta)=\operatorname{Re}\left(\sum_{j} p_{j}(\theta) M_{j} i^{\operatorname{deg}\left(M_{j}\right)}\right), \\
& b(\theta)=\operatorname{Re}\left(\sum_{k} q_{k}(\theta) N_{k} i^{\operatorname{deg}\left(N_{k}\right)}\right),
\end{aligned}
$$

where $M_{j}, N_{k}$ denote monomials of $(1)$, and $p_{j}(\theta), q_{k}(\theta) \in \mathbf{R}\left[e^{i \theta}\right]$. Then,

$$
\begin{aligned}
a(\theta) b(\theta)= & \operatorname{Re}\left[\frac{1}{2} \sum_{j} p_{j}(\theta) M_{j} i^{\operatorname{deg}\left(M_{j}\right)}\left(\sum_{k} q_{k}(\theta) N_{k} i^{\operatorname{deg}\left(N_{k}\right)}+\sum_{k} \bar{q}_{k}(\theta) \bar{N}_{k}(-i)^{\operatorname{deg}\left(\bar{N}_{k}\right)}\right)\right]= \\
= & \operatorname{Re}\left[\sum _ { j , k } \left(\frac{1}{2} p_{j}(\theta) q_{k}(\theta) M_{j} N_{k} i^{\operatorname{deg}\left(M_{j} \cdot N_{k}\right)}+\right.\right. \\
& \left.\left.\frac{1}{2} p_{j}(\theta) \bar{q}_{k}(\theta)(-1)^{\operatorname{deg}\left(\bar{N}_{k}\right)} M_{j} \bar{N}_{k} i^{\operatorname{deg}\left(M_{j} \cdot \bar{N}_{k}\right)}\right)\right],
\end{aligned}
$$

where we have used that $\operatorname{Re}(z) \operatorname{Re}(w)=\operatorname{Re}(z(w+\bar{w})) / 2$, for any $z, w \in \mathbf{C}$. So, the lemma follows.

Proof of Theorem A. First observe that to prove (i) is equivalent to prove that $v_{2 m+1} \in$ $\mathcal{P}_{1}$ (where the trigonometric polynomials involved are constants). To achieve this result we study to which set, $\mathcal{P}_{0}$ or $\mathcal{P}_{1}$, belong the functions appearing in the algorithm of computation of the constants given in [1]. We briefly recall it here. as

Equation (1) can be written in the polar coordinates $r^{2}=z \bar{z}$ and $\theta=\arctan \frac{\operatorname{Im}(z)}{\operatorname{Re}(z)}$,

$$
\frac{d r}{d \theta}=\frac{r^{2} \operatorname{Re}\left(S_{2}(\theta)\right)+r^{3} \operatorname{Re}\left(S_{3}(\theta)\right)+\ldots}{1+r \operatorname{Im}\left(S_{2}(\theta)\right)+r^{2} \operatorname{Im}\left(S_{3}(\theta)\right)+\ldots}=\sum_{k \geq 2} r^{k}(\theta) R_{k}(\theta),
$$

where $S_{k}(\theta)=e^{-i \theta} F_{k}\left(e^{i \theta}, e^{-i \theta}\right)$.

Observe that $S_{k}(\theta)=\sum_{j=0}^{k} f_{k-j, j} e^{i(k-2 j-1) \theta}=\sum_{j=0}^{k} e^{i(k-2 j-1) \theta}\left(-f_{k-j, j}\right) i^{\operatorname{deg}\left(f_{k-j, j}\right)+1}$, because $\operatorname{deg}\left(f_{k-j, j}\right)=1$, for all $j$ and $k$. Hence, it is clear that $\operatorname{Re}\left(S_{k}(\theta)\right) \in \mathcal{P}_{1}$.

The functions $R_{k}(\theta)$ can be computed using the recursive formula for the quotient 
of series given in $[8]$ as

$$
R_{k}(\theta)=\operatorname{Re}\left(S_{k}(\theta)\right)-\sum_{j=1}^{k-2} \operatorname{Im}\left(S_{j+1}(\theta)\right) R_{k-j}(\theta) .
$$

We also have that $\operatorname{Im}\left(S_{j+1}(\theta)\right)=\operatorname{Re}\left(-i S_{j+1}(\theta)\right) \in \mathcal{P}_{0}$. By using Lemma 2, it is easy to prove by induction that $R_{k}(\theta) \in \mathcal{P}_{1}$.

Let $r(\theta, \rho)=\rho+\sum_{j>2} u_{j}(\theta) \rho^{j}$ be the solution of (3) for which $r(0, \rho)=\rho$. Consider the Poincaré map given by $\Pi(\rho)=r(2 \pi, \rho)=\rho+\sum_{j \geq 2} u_{j}(2 \pi) \rho^{j}$. It is well-known that if $\Pi(\rho)$ is not the identity, the first non vanishing term in the power series corresponds to an odd order term. When $u_{2}(2 \pi)=u_{3}(2 \pi)=\ldots=u_{2 m}(2 \pi)=0$ and $u_{2 m+1}(2 \pi) \neq 0$, the $m^{t h}$-Liapunov constant is $v_{2 m+1}=u_{2 m+1}(2 \pi)$.

Recall also that when $u_{2}(2 \pi)=u_{3}(2 \pi)=\ldots=u_{n-1}(2 \pi)=0$, then the functions $u_{j}(\theta)$ are trigonometric polynomials for $j \leq n-1$, and

$$
u_{n}^{\prime}(\theta)=\sum_{k=2}^{n} R_{k}(\theta)\left(\sum_{\mathbf{a} \in D_{n}^{k}} \frac{k !}{a_{1} ! a_{2} ! a_{3} ! \cdots a_{n-1} !} u_{2}^{a_{2}}(\theta) u_{3}^{a_{3}}(\theta) \cdots u_{n-1}^{a_{n}-1}(\theta)\right),
$$

where $D_{n}^{k}$ is the following subset of indices (see [2] for more details):

$$
\begin{aligned}
D_{n}^{k}= & \left\{\mathbf{a}=\left(a_{1}, a_{2}, \cdots, a_{n-1}\right) \in \mathrm{N}^{n-1} \text { such that } a_{1}+\cdots+a_{n-1}=k,\right. \\
& \left.a_{1}+\cdots+j a_{j}+\cdots+(n-1) a_{n-1}=n\right\} .
\end{aligned}
$$

We claim that

$$
u_{n}(\theta)=Q_{n}(\theta)+P_{n}(\theta) \theta
$$

where $Q_{n}(\theta) \in \mathcal{P}_{0}$ and $P_{n}(\theta) \in \mathcal{P}_{1}$. Furthermore,

$$
P_{n}(\theta)=\operatorname{Re}\left(\sum_{l} p_{l}(\theta) M_{l} i^{\operatorname{deg}\left(M_{l}\right)+1}\right), \quad Q_{n}(\theta)=\operatorname{Re}\left(\sum_{l} q_{l}(\theta) M_{l} i^{\operatorname{deg}\left(M_{l}\right)}\right),
$$

where $M_{l}$ denote monomials of $(1), p_{l}(\theta), q_{l}(\theta) \in \mathbf{R}\left[e^{i \theta}\right]$, and have no constant terms.

Observe that if this claim is proved, then Theorem A follows because when $n=$ $2 m+1$, since $v_{2 m+1}=u_{2 m+1}(2 \pi)=\int_{0}^{2 \pi} u_{2 m+1}^{\prime}(\theta) d \theta$, we obtain that $v_{2 m+1} \in \mathcal{P}_{1}$, as we wanted to prove.

We now prove the claim by induction. We have that for $n=2$ :

$$
\begin{aligned}
u_{2}(\theta) & =\operatorname{Im}\left[f_{20} e^{i \theta}-f_{11} e^{-i \theta}-\frac{f_{02}}{3} e^{-3 i \theta}-f_{20}+f_{11}+\frac{f_{02}}{3}\right] \\
& =\operatorname{Re}\left[\left(1-e^{i \theta}\right) f_{20} i+\left(e^{-i \theta}-1\right) f_{11} i+\frac{1}{3}\left(e^{-3 i \theta}-1\right) f_{02} i\right] \in \mathcal{P}_{0}
\end{aligned}
$$

Suppose that the claim is true for $u_{j}(\theta)$ with $j=2, \ldots, n-1$. Since the functions $u_{j}$, for $j \leq n-1$, are trigonometric polynomials (that is, they do not contain terms with the factor $\theta$ ), applying the induction hypothesis we get that $u_{j}(\theta) \in \mathcal{P}_{0}$, for 
$j \leq n-1$. Since $u_{n}^{\prime}(\theta)$ is obtained from (4), considering Lemma 2 and the structure of $R_{k}(\theta)$, we obtain that $u_{n}^{\prime}(\theta) \in \mathcal{P}_{1}$.

We distinguish in $u_{n}^{\prime}(\theta)$ two types of terms: those of the form $\operatorname{Re}\left(C M i^{\operatorname{deg}(M)+1}\right)$, and those of the form $\operatorname{Re}\left(C e^{i \alpha \dot{\theta}} M i^{\operatorname{deg}(M)+1}\right)$, with $C, \alpha \in \mathbf{R}, \alpha \neq 0$, and $M$ a monomial of (1).

By integrating between 0 and $\theta$, the terms of the first form are transformed into

$$
\operatorname{Re}\left(C M i^{\operatorname{deg}(M)+1}\right) \theta
$$

and so they are terms of the form $p(\theta) \theta$, with $p(\theta) \in \mathcal{P}_{1}$, as we wanted to prove.

The integration of the second form terms between 0 and $\theta$ leads to expressions like

$$
\begin{aligned}
& \operatorname{Re}\left(\frac{C}{i \alpha} e^{i \alpha \theta} M i^{\operatorname{deg}(M)+1}-\frac{C}{i \alpha} M i^{\operatorname{deg}(M)+1}\right)= \\
= & \operatorname{Re}\left(\frac{-C}{\alpha} e^{i \alpha \theta} M i^{\operatorname{deg}(M)+2}-\frac{-C}{\alpha} M i^{\operatorname{deg}(M)+2}\right)= \\
= & \operatorname{Re}\left(\frac{C}{\alpha} e^{i \alpha \theta} M i^{\operatorname{deg}(M)}-\frac{C}{\alpha} M i^{\operatorname{deg}(M)}\right),
\end{aligned}
$$

and so they belong to $\mathcal{P}_{0}$, as we wanted to prove. Hence, the claim is proved and, as a consequence, the proof of (i) is finished.

The proof of (ii) is similar and we just establish the differences. Assume that (1) has a center at the origin. Consider (1) expressed in polar coordinates. The period function, which gives the period of the orbit of (2) for which $r(0, \rho)=\rho$ can be expressed as

$$
\begin{aligned}
P(\rho) & =2 \pi+\sum_{k \geq 1} \int_{0}^{2 \pi} H_{k}(\theta) r(\theta, \rho)^{k} d \theta= \\
& =2 \pi+\sum_{k \geq 1} t_{k}(2 \pi) \rho^{k},
\end{aligned}
$$

where the functions $H_{k}(\theta)$ can be calculated using the recursive formula

$$
H_{k}(\theta)=-\sum_{j=1}^{k} \operatorname{Im}\left(S_{j+1}(\theta)\right) H_{k-j}(\theta),
$$

and the functions $t_{k}(\theta)$ satisfy the following recurrence:

(6) $t_{k}^{\prime}(\theta)=H_{1}(\theta) u_{k}(\theta)+\sum_{m=2}^{k} H_{m}(\theta)\left[\sum_{\mathbf{a} \in D_{k}^{m}} \frac{m !}{a_{1} ! a_{2} ! a_{3} ! \cdots a_{k-1} !} u_{2}^{a_{2}}(\theta) u_{3}^{a_{3}}(\theta) \cdots u_{k-1}^{a_{k-1}}(\theta)\right]$,

see again [2] for more details.

It is well-known that the first non vanishing term in the power series of $P(\rho)$ corresponds to an even order term. So, if $t_{2}(2 \pi)=t_{3}(2 \pi)=\ldots=t_{2 m-1}(2 \pi)=0$ and $t_{2 m}(2 \pi) \neq 0$, the $m^{t h}$ period constant is $P_{2 m}=t_{2 m+1}(2 \pi)$.

In the above notation, the proof of (ii) follows from similar arguments than in (i), but considering the recursive formula (5) instead of (3), and (6) instead of (4). 
3. THE ANALYTIC-NUMERICAL METHOD WITH APPLICATIONS.

Here we present a method to compute the general formula of the constants. Let us suppose, for instance, that we want to find the expression for $v_{2 m+1}$. We proceed as follows:

Step 1. By using Theorems 1, A and the Appendix, we list all the monomials involved in $v_{2 m+1}$. That is, we write $v_{2 m+1}$ as a linear function of products of basic monomials (see Remark 4 in the Appendix).

Step 2. Once the monomials are listed, we look for all the undeterminated coefficients by computing the constant for some particular systems. To do this, we use the RungeKutta-Fehlberg 7-8 method to calculate the Poincaré return map. Afterwards, we apply the Richardson's extrapolation method in order to reduce the error.

The above procedure will be followed in the next subsection to compute the expression of $v_{5}$ for a general system of type (1). Step 2 can also be used to obtain numerically the $m^{\text {th }}$ Liapunov constant for a particular differential equation of type (1). This has already been done in [5], but here we also study the behaviour of the round-off errors. In subsection 3.2 we present how to compute the third Liapunov constant, $v_{7}$, for a quadratic system.

First of all, let us describe Step 2 more carefully in the case of the computation of $v_{5}$.

It is known that when $v_{3}=0$, the Poincaré map $\Pi(x)$ near the origin is given by:

$$
\Pi(x)-x=v_{5} x^{5}+o\left(x^{5}\right),
$$

where $x$ is the first coordinate of a point on the semi-axis $\{(x, 0): x>0\}$. Then,

$$
F(x)=\frac{\Pi(x)-x}{x^{5}}=v_{5}+o(x)=v_{5}+a_{1} x^{r_{1}}+a_{2} x^{r_{2}}+\ldots,
$$

where $1 \leq r_{1}<r_{2}<r_{3}<\ldots$

The constant $v_{5}$ could be approximated by a direct computation of $F(x)$ for $x$ small enough, but the factor $x^{r_{i}}$ might not be small enough near $x=0$. We can obtain a better precision by increasing the powers $r_{i}$. This can be done by the Richardson's extrapolation method, next described.

From a sequence of values of $F(x)$, namely $F\left(x_{1}\right), F\left(x_{2}\right), \ldots, F\left(x_{m}\right)$, such that $x_{i+1}=q x_{i}, x_{1}=x, i=1, \ldots, m-1$ and $q>1$, we define:

$$
\left\{\begin{array}{l}
F_{1}(x)=F(x) \\
F_{j+1}(x)=F_{j}(x)+\frac{F_{j}(x)-F_{j}(q x)}{q^{\tau_{j}}-1}, \quad \text { for all } j \geq 1 .
\end{array}\right.
$$

Then, it can be proved that:

$$
F_{k}(x)=v_{5}+a_{k}^{(k)} x^{r_{k}}+a_{k+1}^{(k)} x^{r_{k+1}}+\ldots, \quad k \leq m .
$$

Therefore, $F_{k}(x)$ is a better approximation for $v_{5}$ than $F(x)$, for $x$ small enough (see [11] for more details). 
TABLE 2

\begin{tabular}{|c|c|}
\hline Degree & Monomials of quasi-degree 4 and weight 0. \\
\hline \hline 1 & $O$ \\
\hline 2 & $D \bar{D}, F \bar{F}, G \bar{G}, D F, A \bar{I}, A J, B I, B \bar{J}, C H, C \bar{K}, E^{2}, E \bar{E}$ \\
\hline 3 & $A^{2} F, A^{2} \bar{D}, B^{2} D, B^{2} \bar{F}, A \bar{B} F, A \bar{B} \bar{D}, A C D$, \\
& $A C \bar{F}, B \bar{C} F, B \bar{C} \bar{D}, A \bar{C} G, B C \bar{G} A \bar{A} E, B \bar{B} E, C \bar{C} E, A B E, A B \bar{E}$ \\
\hline 4 & $A^{3} C, A^{2} \bar{B} C, A \bar{B}^{2} C, B^{3} \bar{C}, A^{2} \bar{A}^{2}, A B \bar{A} \bar{B}$, \\
& $A C \bar{A} \bar{C}, A^{2} \bar{A} B, B^{2} \bar{B}^{2}, B C \bar{B} \bar{C}, A B^{2} \bar{B}, C^{2} \bar{C}^{2}, A B \bar{C}, A^{2} B^{2}$ \\
\hline
\end{tabular}

The images $F\left(x_{1}\right), \ldots, F\left(x_{m}\right)$ are obtained by using the Runge-Kutta-Fehlberg method of orders 7 and 8 , with tolerance $\epsilon_{1}=10^{-13}$, and an initial step $h_{i}=10^{-5}$, with maximum and minimum steps $h_{M}=10^{-1}$ and $h_{m}=10^{-16}$, respectively, and a precision of $10^{-16}$. Observe that this is a three-parametric method, with parameters $x, q$ and $m$.

3.1. Computation of the second Liapunov constant. In this subsection, by using the above procedure, we compute the expression of the second Liapunov constant $v_{5}$. This gives the following well-known result (see [1], [2], [4], [7], [9] and $[15]$.

Theorem 3. Consider the equation $\dot{z}=i z+F(z, \bar{z})$ with $F_{2}(z, \bar{z})=A z^{2}+B z \bar{z}+$ $C \bar{z}^{2}, F_{3}(z, \bar{z})=D z^{3}+E z^{2} \bar{z}+F z \bar{z}^{2}+G \bar{z}^{3}, F_{4}(z, \bar{z})=H z^{4}+I z^{3} \bar{z}+J z^{2} \bar{z}^{2}+K z \bar{z}^{3}+L \bar{z}^{4}$ and $F_{5}(z, \bar{z})=M z^{5}+N z^{4} \bar{z}+O z^{3} \bar{z}^{2}+P z^{2} \bar{z}^{3}+Q z \bar{z}^{4}+R \bar{z}^{5}$. Then:

(i) $v_{3}=2 \pi[\operatorname{Re}(E)-\operatorname{Im}(A B)]$.

(ii) $v_{5}=\frac{\pi}{3}\left[6 \operatorname{Re}(O)+\operatorname{Im}\left(3 E^{2}-6 D F+6 A \bar{I}-12 B I-6 B \bar{J}-8 C H-2 C \bar{K}\right)+\right.$ $\operatorname{Re}\left(-8 C \bar{C} E+4 A C \bar{F}+6 A \bar{B} F+6 B \bar{C} F-12 B^{2} D-4 A C D-6 A \bar{B} \bar{D}+10 B \bar{C} \bar{D}+4 A \bar{C} G+\right.$ $\left.2 B C \bar{G})+\operatorname{Im}\left(6 A \bar{B}^{2} C+3 A^{2} B^{2}-4 A^{2} \bar{B} C+4 \bar{B}^{3} C\right)\right]$.

By using the Appendix, we start listing the monomials satisfying Theorem 1. They are given in Table 2.

By Theorem A, the real monomials of even degree $\left(D \bar{D}, F \bar{F}, G \bar{G}, E \bar{E}, A^{2} \bar{A}^{2}\right.$, $\left.A B \bar{A} \bar{B}, A C \bar{A} \bar{C}, B^{2} \bar{B}^{2}, B C \bar{B} \bar{C}, C^{2} \bar{C}^{2}\right)$ do not appear in $v_{5}$. Moreover, imposing that $v_{3}=0$, i.e. $\operatorname{Re} E=\operatorname{Im}(A B)$, we can consider that $\operatorname{Im}(A B C \bar{C})=C \bar{C} \operatorname{Im}(A B)=$ $\operatorname{Re}(C \bar{C} E)$ and so, the monomials $A B E, A B \bar{E}, A^{2} \bar{A} B, A B^{2} \bar{B}$ and $A B C \bar{C}$ can be eliminated. On the other hand, since the systems of type $\dot{z}=i z+A z^{2}+D z^{3}$ are isochronous centers, we can also deduce that the monomial $A^{2} \bar{D}$ does not appear. Taking all this into account, we end Step 1 and we have that

$$
\begin{gathered}
v_{5}=\frac{\pi}{3}\left[\alpha_{1} \operatorname{Re} O+\operatorname{Im}\left(\alpha_{2} D F+\alpha_{3} A \bar{I}+\alpha_{4} A J+\alpha_{5} B I+\alpha_{6} B \bar{J}+\alpha_{7} C H+\alpha_{8} C \bar{K}+\alpha_{9} E^{2}\right)+\right. \\
\operatorname{Re}\left(\alpha_{10} A^{2} F+\alpha_{11} B^{2} D+\alpha_{12} B^{2} \bar{F}+\alpha_{13} A \bar{B} F+\alpha_{14} A \bar{B} \bar{D}+\alpha_{15} A C D+\alpha_{16} A C \bar{F}+\right. \\
\left.\alpha_{17} B \bar{C} F+\alpha_{18} B \bar{C} \bar{D}+\alpha_{19} A \bar{C} G+\alpha_{20} B C \bar{G}+\alpha_{21} A \bar{A} E+\alpha_{22} B \bar{B} E+\alpha_{23} C \bar{C} E\right)+ \\
\left.\operatorname{Im}\left(\alpha_{24} A^{3} C+\alpha_{25} A^{2} \bar{B} C+\alpha_{26} A \bar{B}^{2} C+\alpha_{27} B^{3} \bar{C}+\alpha_{28} A^{2} B^{2}\right)\right] .
\end{gathered}
$$


TABLE 3

Determination of the coefficients of $v_{5}$.

\begin{tabular}{|c|c|c|}
\hline & $\begin{array}{c}\text { Equation } \dot{z}=i z+F(z, \bar{z}) \\
\text { where } F(z, \bar{z}) \text { is: }\end{array}$ & Linear equation in $\alpha_{k}$ \\
\hline 1 & $z^{3} \bar{z}^{2}$ & $\alpha_{1}=6$ \\
\hline 2 & $z^{3}-i z \bar{z}^{2}$ & $-\alpha_{2}=6$ \\
\hline 3 & $z^{2}-i z^{3} \bar{z}$ & $\alpha_{3}=6$ \\
\hline 4 & $z^{2}+i z^{2} \bar{z}^{2}$ & $\alpha_{4}=0$ \\
\hline 5 & $z \bar{z}-i z^{3} \bar{z}$ & $-\alpha_{5}=12$ \\
\hline 6 & $z \bar{z}+i z^{2} \bar{z}^{2}$ & $-\alpha_{6}=6$ \\
\hline 7 & $\bar{z}^{2}-i z^{4}$ & $-\alpha_{7}=8$ \\
\hline 8 & $\bar{z}^{2}+i z \bar{z}^{3}$ & $-\alpha_{8}=2$ \\
\hline 9 & $z^{2}+z \bar{z}^{2}$ & $\alpha_{10}=0$ \\
\hline 10 & $z \bar{z}-z^{3}$ & $-\alpha_{11}=12$ \\
\hline 11 & $z \bar{z}+z \bar{z}^{2}$ & $\alpha_{12}=0$ \\
\hline 12 & $z^{2}+z \bar{z}+z \bar{z}^{2}$ & $\alpha_{10}+\alpha_{12}+\alpha_{13}=6$ \\
\hline 13 & $z^{2}+z \bar{z}-z^{3}$ & $-\alpha_{11}-\alpha_{14}=18$ \\
\hline 14 & $z^{2}+\bar{z}^{2}-z^{3}$ & $-\alpha_{15}=4$ \\
\hline 15 & $z^{2}+\bar{z}^{2}+z \bar{z}^{2}$ & $\alpha_{10}+\alpha_{16}=4$ \\
\hline 16 & $z \bar{z}+\bar{z}^{2}+z \bar{z}^{2}$ & $\alpha_{12}+\alpha_{17}=6$ \\
\hline 17 & $z \bar{z}+\bar{z}^{2}+z^{3}$ & $\alpha_{11}+\alpha_{18}=-2$ \\
\hline 18 & $z^{2}+\bar{z}^{2}+\bar{z}^{3}$ & $\alpha_{19}=4$ \\
\hline 19 & $\overline{z \bar{z}+\bar{z}^{2}+\bar{z}^{3}}$ & $\alpha_{20}=2$ \\
\hline 20 & $i z^{2}+\bar{z}^{2}$ & $-\alpha_{24}=0$ \\
\hline 21 & $z \bar{z}+i \bar{z}^{2}$ & $-\alpha_{27}=4$ \\
\hline 22 & $z^{2}+i z \bar{z}+(1+i) z^{2} \bar{z}$ & $\alpha_{21}+\alpha_{22}+2 \alpha_{9}=6$ \\
\hline 23 & $z^{2}+(1+i) z \bar{z}+(1+i) z^{2} \bar{z}$ & $2 \alpha_{9}+\alpha_{21}+2 \alpha_{22}+2 \alpha_{28}=12$ \\
\hline 24 & $(1+i) z^{2}+z \bar{z}+(1+i) z^{2} \bar{z}$ & $2 \alpha_{9}+2 \alpha_{21}+\alpha_{22}+2 \alpha_{28}=12$ \\
\hline 25 & $z^{2}+(1+i) z \bar{z}+z^{2} \bar{z}$ & $\alpha_{21}+2 \alpha_{22}+2 \alpha_{28}=6$ \\
\hline 26 & $z^{2}-i z \bar{z}+\bar{z}^{2}-z^{2} \bar{z}$ & $-\alpha_{21}-\alpha_{22}-\alpha_{23}+\alpha_{25}+\alpha_{27}=0$ \\
\hline 27 & $i z^{2}+z \bar{z}+\bar{z}^{2}+z^{2} \bar{z}$ & $\alpha_{21}+\alpha_{22}+\alpha_{23}-\alpha_{24}+\alpha_{26}=-2$ \\
\hline 28 & $i z^{2}+i z \bar{z}+\bar{z}^{2}$ & $-\alpha_{24}+\alpha_{25}-\alpha_{26}-\alpha_{27}=-6$ \\
\hline
\end{tabular}

Now we must search for the undetermined coefficients $\alpha_{j}$, for $j=1, \ldots, 28$.

From formula (4) we can deduce that the coefficients $\alpha_{j}$ of the monomials of $v_{5}$ have a common factor $\pi$. We also introduce a handling which permits a higher reliability on the numerical results, since we have observed that taking a system with integer coefficients and multiplying by $\frac{3}{\pi}$ the numerical approximation of the constant obtained from Step 2, we get a result very close to an integer number. In fact, we obtain numbers $v$ such that there exist $n \in \mathbf{Z}$ satisfying $|v-n|<10^{-3}$. Then, it is clear which is the integer result we must consider to be the correct one. For instance, if we take System 1 of Table 3 and we apply to it the Step 2 of our method, we have that $\frac{3}{\pi} v_{5} \approx 5.9999984$. So we consider that $\frac{3}{\pi} v_{5}=6$.

We choose 28 differential equations for which $v_{3}=0$ and for which many of the monomials appearing in $v_{5}$ vanish. We compute the value of the constant applying the numerical method explained above and we reach a linear system of 28 equations where each component of the independent vector is the result of one of the 28 numerical experiments, rounded-off to the closer integer number. In Table 3, we show the differential equations choosen and the linear equations derived from them. 
Solving the linear system, we obtain the coefficients of the expression of Theorem 3 (ii). Of course, it coincides with the results obtained in the previous works, although

this procedure cannot be strictly considered as a proof. A similar idea could be used to find the period constants.

3.2. Computation of third Liapunov constant for a particular system. In this example, we apply the numerical method to compute $v_{7}$ for the particular quadratic system

$$
\left\{\begin{array}{l}
\dot{x}=-y+x^{2}+2 x y \\
\dot{y}=x+x^{2}+3 x y-y^{2}
\end{array}\right.
$$

for which it is known (see Remark 4 in the Appendix) that $v_{3}=v_{5}=0$ and $v_{7}=$ $\frac{25 \pi}{32} \approx 2.45436926$.

We are going to show how we obtained an approximation of this value from the numerical procedure explained at the beginning of this section. The final value obtained from that procedure, after fixing $x, q$ and $m$, will be called $v_{7}(x, q, m)$.

We consider $x \in I_{x}=[0.01,0.065], q \in I_{q}=[1.075,1.425]$ and $m \in I_{m}=$ $\{4,5,6,7\}$. Then, with the values of $v_{7}(x, q, m)$ obtained for $(x, q, m) \in I_{x} \times I_{q} \times I_{m}$, we make a simple statistical estimation of the value of $v_{7}$.

The estimator we consider is the sample mode. The main reason is that we believe that when the $(x, q, m)$-process works, it gives good approximations of $v_{7}$; however, when it does not work (for instance, because of the numerical unstability), the error can be big and often with the same sign. This is a definitive argument against the consideration of the mean and the median in our problem.

To display the accuracy of this example, we give some graphics of the frequencies (see Figure 1), considering from graphic to graphic one more digit of the values. In this way, it can be observed that (for this example) the method adjustes statistically up to the third digit. In the third graphic (corresponding to the fourth digit, see Figure 1.c), we can appreciate a great dispersion of the values, which indicates the reliability of the method just only up to the third digit, with a relative error of $\delta \approx \frac{5}{2.45} 10^{-3} \approx 2 \cdot 10^{-3}$.

Obviously, this numerical conclusion is only valid for this example, but the procedure can be applied to any other system.

APPENDIX: FINITENESS OF BASIC MONOMIALS. In this appendix we prove the following result.

Proposition B The number of basic monomials associated to system (1) with $F(z, \bar{z})$ polynomial is finite.

REMARK 4. Denote by $\mathcal{B}$ the finite set of basic monomials associated to a polynomial system (1). Observe that the above result implies that the Liapunov constants are real-valued polynomials in the ring $\mathrm{C}[\mathcal{B}]$. For instance, if we consider system $\dot{z}=i z+f_{20} z^{2}+f_{11} z \bar{z}+f_{02} \bar{z}^{2}$. The basic monomials associated to it are $B_{1}=f_{20} \bar{f}_{20}$, 
$B_{2}=f_{11} \bar{f}_{11}, B_{3}=f_{02} \bar{f}_{02}, B_{4}=f_{20} f_{11}, B_{5}=f_{20}^{3} f_{02}, B_{6}=\bar{f}_{11}^{3} f_{02}, B_{7}=f_{20}^{2} \bar{f}_{11} f_{02}$, $B_{8}=f_{20} \bar{f}_{11}^{2} f_{02}$ and their conjugates. Furthermore, applying also Theorems 1 and $A$ we get that

$$
\begin{aligned}
& v_{3}=\alpha_{1} \operatorname{Im} B_{4} \\
& v_{5}=\operatorname{Im} F_{0} \\
& v_{7}=\sum_{i=1}^{3} B_{i} \operatorname{Im} F_{i}+\operatorname{Im}\left(B_{4} F_{4}+\bar{B}_{4} F_{5}\right),
\end{aligned}
$$

where $F_{i}=F_{i}\left(B_{5}, B_{6}, B_{7}, B_{8}\right)$ are linear functions in all the variables.

Indeed, it is a known fact that for quadratic systems:

$$
\begin{aligned}
& v_{3}=-2 \pi \operatorname{Im}\left(B_{4}\right), \\
& v_{5}=-\frac{2 \pi}{3} \operatorname{Im}\left(2 B_{7}-3 B_{8}-2 B_{6}\right), \\
& v_{7}=-\frac{5 \pi}{4}\left(B_{2}-B_{3}\right) \operatorname{Im}\left(2 B_{8}+B_{6}\right) .
\end{aligned}
$$

We will use the next technical result to prove Proposition B.

Lemma 5. Consider $\alpha_{1}, \alpha_{2}, \ldots, \alpha_{s} \in \mathbf{Z}$. Then, there exists a positive integer $m$, $0 \leq m \leq s$, and a reordenation $\left\{\beta_{1}, \ldots, \beta_{s}\right\}$ of the numbers $\alpha_{1}, \ldots, \alpha_{s}$ such that

$$
\sum_{i=1}^{m} \beta_{i} \equiv 0 \quad(\bmod s)
$$

Proof. Consider $S_{k}:=\sum_{i=1}^{k} \beta_{i}$, for all $k \leq s$. If $S_{k} \equiv S_{j} \quad(\bmod s)$ for some $k \neq j$ (let us suppose $k>j)$, then $S_{k}-S_{j}=\alpha_{j+1}+\ldots+\alpha_{k} \equiv 0(\bmod s)$, and so the lemma would be fulfilled. Otherwise, $S_{1}, S_{2}, \ldots, S_{s}$ belong to different classes in $\mathbf{Z}_{s}$ and therefore, one of then must be $\overline{0} \in \mathbf{Z}_{s}$. Then, the lemma also follows.

Proof of Proposition B. Denote by $B=C_{1}^{\alpha_{1}} \cdots C_{n}^{\alpha_{n}}$, a basic monomial of (1), where $C_{1}, \ldots, C_{n}$, are the coefficients of the system and suppose that $\left|\mathrm{w}\left(C_{i}\right)\right|=i$, for all $i$. In fact, $C_{i}$ is a generic label representing any coefficient of (1) of weight $\pm i$, while $\alpha_{i}$ is the number of coefficients taking into account the repetitions (in a basic monomial of degree greater than two, there cannot coexist coefficients of weight $i$ and $-i$ ). Then, Proposition $\mathrm{B}$ follows if we are able to prove that equation

$$
\alpha_{1}+2 \alpha_{2}+\ldots+n \alpha_{n}=0
$$

has finitely many minimal solutions, where we say that a solution $\alpha:=\left(\alpha_{1}, \ldots, \alpha_{n}\right) \in$ $\mathrm{Z}^{n}$ is a minimal solution of $(7)$ if it does not exist any other solution $\left(\beta_{1}, \ldots, \beta_{n}\right)$ such that $\left|\beta_{i}\right| \leq\left|\alpha_{i}\right|$ and $\operatorname{sign}\left(\beta_{i}\right)=\operatorname{sign}\left(\alpha_{i}\right)$, for all $i=1, \ldots, n$.

In particular, the finiteness of minimal solutions will be proved if we prove that any minimal solution $\alpha$ of $(7)$ satisfies that $\left|\alpha_{i}\right| \leq n$, for all $i=1, \ldots, n$. 
Suppose that $\alpha_{k}>n$ for some $k \in\{1, \ldots, n\}$. First, we will see that this implies that $\alpha_{j} \geq-n$ for any $j$. Otherwise, if for some $j$ we have $\alpha_{j}<-n$, we can write $\alpha_{k}=j+N_{k}$ and $\alpha_{j}=-k-N_{j}$, where $N_{k}$ and $N_{j}$ are positive numbers. Then,

$$
\begin{aligned}
\alpha= & \left(\alpha_{1}, \ldots, \alpha_{j}, \ldots, \alpha_{k}, \ldots, \alpha_{n}\right)= \\
& \left(\alpha_{1}, \ldots,-N_{j}, \ldots, N_{k}, \ldots, \alpha_{n}\right)+(0, \ldots, 0,-k, \ldots, j, 0, \ldots, 0),
\end{aligned}
$$

and this situation breaks the property of minimality of $\alpha$.

Now, we keep the assumption that $\alpha_{k}>n$ for some $k \in\{1, \ldots, n\}$. Since $\alpha$ must be a solution of (7), we deduce that there must exist $\alpha_{j_{1}}, \ldots, \alpha_{j_{r}}$, all of them negative, such that $\sigma_{k}:=\sum_{i=1}^{r} j_{i} \alpha_{j_{i}}<-k n$. We can write

$$
\begin{aligned}
& \left(-\alpha_{j_{1}}\right) \quad\left(-\alpha_{j_{r}}\right) \\
& \sigma_{k}=-\left(j_{1}+\ldots+j_{1}+\ldots+j_{r}+\ldots+j_{r}\right) .
\end{aligned}
$$

Since $j_{i} \leq n$ for all $i$ and $\sigma_{k}<-k n$, the number of terms involved in the last expression of $\sigma_{k}$ is greater or equal than $k$. Then, we choose any string of $k$ terms in $\sigma_{k}$ and we call it $\sigma_{k}^{\prime}$. It is obvious that

$$
\sigma_{k}^{\prime}=\sum_{i=1}^{r} j_{i} \alpha_{j_{i}}^{\prime}, \text { with } 0 \geq \alpha_{j_{i}}^{\prime} \geq \alpha_{j_{i}} \text { for all } i .
$$

Then, since $\sigma_{k}^{\prime}$ is a string of length $k$, we can apply Lemma 5 to it and deduce that it contains a partial sum, $\sigma_{k}^{\prime \prime}$, such that $\sigma_{k}^{\prime \prime} \equiv 0(\bmod k)$. Then, if we write $\sigma_{k}^{\prime \prime}=\sum_{i=1}^{r} j_{i} \alpha_{j_{i}}^{\prime \prime}=-k \mu$, with $0<\mu \leq n$, we can construct the following solution $\beta:=\left(\beta_{1}, \ldots, \beta_{n}\right)$ of $(7)$ :

$$
\beta_{i}= \begin{cases}\alpha_{j_{i}}{ }^{\prime \prime} & \text { if } i \in\left\{j_{1}, \ldots, j_{r}\right\}, \\ \mu & \text { if } i=k, \\ 0 & \text { if } i \notin\left\{k, j_{1}, \ldots, j_{r}\right\} .\end{cases}
$$

From their definition, it is clear that $0 \geq \alpha_{j_{i}}{ }^{\prime} \geq \alpha_{j_{i}}$, for all $i \in\{1, \ldots, r\}$. On the other hand, $0<\mu \leq n<\alpha_{k}$. Then, $\beta$ is a solution which breaks again the minimality of $\alpha$ and gives the contradiction we were searching for. So, the proposition is proved.

\section{REFERENCES}

[1] A.A. Andronov, E.A. Leontovich, I.I. Gordon, A.G. MaIER, Theory of Bifurcations of Dynamic Systems on a Plane, John Wiley \& Sons, New York, Chapter IX, 1973.

[2] A. Cima, A. Gasull, V. Mañosa, F. Mañosas, Algebraic properties of the Liapunov and period constants, Rocky Mountain J. Math., to appear.

[3] C. Chicone, M. JACoBs, Bifurcation of critical periods for plane vector fields, Trans. Amer. Math. Soc., 312 (1989), pp. 433-486.

[4] W.W. Farr, Li Chengzhi, I.S. Labouriau, W.F. LangFord, Degenerate Hopf bifurcation formulas and Hilbert's 16th problem, SIAM J. Math. Anal., 20 (1989), pp. 13-29.

[5] A. Gasull, A. Guillamon, Li Chengzhi, Zhang Zhifen, Study of perturbed Lotka-Volterra systems via Abelian integrals, J. Math. Anal. Appl., to appear. 
[6] A. Gasull, A. Guillamon, V. Mañosa, An explicit expression of the first Liapunov and period constants with applications, preprint (1995).

[7] A. Gasuld, R. Prohens, Effective computation of the first Lyapunov quantities for a planar differential equation, preprint (1994).

[8] I.S. Gradshteyn, I.M. Ryzhik, Table of Integrals, Series and Products, Academic Press Inc., New York, p. 14, 1980.

[9] F. GöbBer, K.D. Willamowski, Liapunov approach to multiple Hopf bifurcation, J. Math. Anal. Appl., 71 (1979), pp. 333-350.

[10] B. HAssaRD, Y.H. WAN, Bifurcation formulae derived from Center Manifold Theory, J. Math. Anal. Appl., 63 (1978), pp. 297-312.

[11] D. Kincaid, W. Cheney, Numerical Analysis, Brooks/Cole Publishing Company, Pacific Grove, California, pp. 436-441, 1991.

[12] LiN YiPING, Li JI-BiN, Normal form of planar autonomous system and periodic critical point, Acta Math. Sinica, 34 (1991), pp. 490-501 (in Chinese).

[13] LiU YI-Rong, Li Ji-Bin, Theory of values of singular point in complex autonomous differential systems, Science in China (Ser. A), 33 (1990), pp. 10-23.

[14] N.G. Lloyd, J.M. PeARson, REDUCE and the bifurcation of limit cycles, J. Symbolic Computation, 9 (1990), pp. 215-224.

[15] E.J. Ponce-NúÑ̃ez, E. Gamero, Generating Hopf bifurcation formulae with MAPLE, Internat. Ser. Numerical Math., 97 (1991), pp. 295-298.

[16] V.G. RomanovskiI, Calculation of Lyapunov numbers in the case of two pure imaginary roots, Differential Equations, 29 (1993), pp. 782-784.

[17] K.S. SibiRskit, Algebraic Invariants for Differential Equations and Matrices, Kshinev, Shtïnt-sa, 1976 (in Russian).

[18] H. ŻOEA̧DEK, Quadratic systems with center and their perturbations, J. Differential Equations, 109 (1994), pp. 223-273. 
This figure is not available in the PostScript version of the paper. There is a file FIGURE.ZIP in this directory. Thank you.

FIG. 1. Frequencies for the value of $v_{7}$ increasing the number of digits of precision. 